\title{
Spatiotemporal aspects of flood exposure in Switzerland
}

\author{
Veronika Röthlisberger ${ }^{1,2, a}$, Andreas Zischg ${ }^{1,2}$ and Margreth Keiler ${ }^{2}$ \\ ${ }^{1}$ University of Bern, Institute of Geography, Hallerstrasse 12, CH-3012 Bern, Switzerland \\ ${ }^{2}$ University of Bern, Oeschger Centre for Climate Change Research, Falkenplatz 16, CH-3012 Bern, Switzerland
}

\begin{abstract}
While flood hazard mapping in Switzerland is close to completion, only a limited number of studies have been specifically conducted on exposure and vulnerability. We fill this knowledge gap by conducting a nation-wide study of flood exposure of buildings in Switzerland. Therefore, we generate a country-wide comprehensive and homogenous data set of polygons of residential buildings and their period of construction and overlay these building polygons with compiled and harmonized flood hazard maps provided by the Swiss cantons. In this paper we present first results of spatiotemporal analyses, namely the evolution of exposure from 1919 to 2012. Surprising is the increase in the share of exposure of new constructed buildings since the 1980s which contradicts the indented effects of the Swiss flood risk management strategies and calls for further investigations.
\end{abstract}

\section{Introduction}

Floods are one main hazard type in the world-wide event and loss database on natural disasters. The data highlight an increasing number of reported events, of people affected and of economic losses, but a decreasing number of reported fatalities since around 1900, especially in the most developed countries [1, 2]. The IPCC [3] identified exposure and vulnerability as key determiners of disaster risk. Furthermore, there is high confidence that "increasing exposure of people and economic assets has been the major cause of long-term increases in economic losses from weather- and climaterelated disasters" [3:7]. Being aware of these important drivers - beside the challenge of climate change efficient flood risk management strategies are strongly related to the availability of data and the assessment of elements at risk [4]. Investigations on the long-term evolution of exposure and the effects on flood risk is still rare, and most studies focus only on the local scale with individual case studies. However, the first nation-wide projects indicate important insights $[5,6]$.

In line with international standards, the main components of risk assessments in Switzerland are hazard mapping, exposure and vulnerability analyses. While flood hazard mapping in Switzerland is close to completion [7], only a limited number of studies have been specifically conducted on exposure and vulnerability. Several Swiss cantons have investigated their exposure and/or vulnerability to floods, and many insurance companies continue to use hazard maps for the risk management of their portfolio. However, these studies are either limited in space (e.g. to the area of a canton) or in content (e.g. based only on the assets insured by a single company), and findings are rarely published. An exception is a publication by SwissRe with countrywide sums on insured property losses in Switzerland for different return periods based on their inhouse flood risk model [8]. Yet, the description of the model and the results are rather generic, without data about elements at risk. To summarise, there is a lack of nation-wide exposure analysis to floods scenarios. Furthermore, all exposure or risk assessments are based on the analysis of the current status, which neglects the long-term evolution of exposure or risk. Consequently, the analysis regarding the long-term evolution of the key driver of exposure is missing in Switzerland too. Both analyses are very important to gain insights into the effectiveness of risk management strategies (such as land-use planning, which has been applied in Switzerland since the 1970s) and to design future flood risk management strategies based on these insights In this paper, we focus on following main questions and discuss the possible relation to flood risk management:

- How have residential buildings exposed to flood evolved temporally?

- Do exposure ratios at the different hazard levels mapped show different temporal patterns?

\section{Data and Methods}

To analyse flood exposure, we link data on flood hazardous areas with spatially explicit information on buildings. In the following sections, the data sets and the methods used are described.

\footnotetext{
a Corresponding author: veronika.roethlisberger@giub.unibe.ch
} 


\subsection{Flood hazard maps}

For the determination of flood endangered areas, we use flood hazard maps provided by the Swiss cantons and elaborated according to national guidelines [9, 10]. Flood hazard is thereby defined by a combination of intensity and probability of events (see Fig. 1) with thresholds in terms of intensity at $0.5 \mathrm{~m}$ (water depth) or $0.5 \mathrm{~m} / \mathrm{s}^{2}$ (water depth $\mathrm{x}$ velocity), for the differentiation between 'weak' and 'medium' intensity, and at $2 \mathrm{~m}$ or $2 \mathrm{~m} / \mathrm{s}^{2}$, respectively, to differentiate between 'medium' and 'strong' intensity. The probabilities of occurrence investigated correspond to return periods of 30 (high), 100 (medium), 300 (slight) and above 300 (very slight) years, respectively. The spatial representation and combination of events of different return periods lead to the mapping of five hazard classes: 'high' (red coloured areas), 'medium' (blue), 'low' (yellow), 'residual' (yellow-white striped) and 'no or negligible' (white) threat (see Fig. 1). In this paper, we focus on three classes - 'high', 'medium' and 'low' - i.e. on areas endangered by flood events up to a return period of 300 years. The flood hazard maps as of June 2015 are harmonized and compiled in a geodatabase by the Mobiliar insurance company.

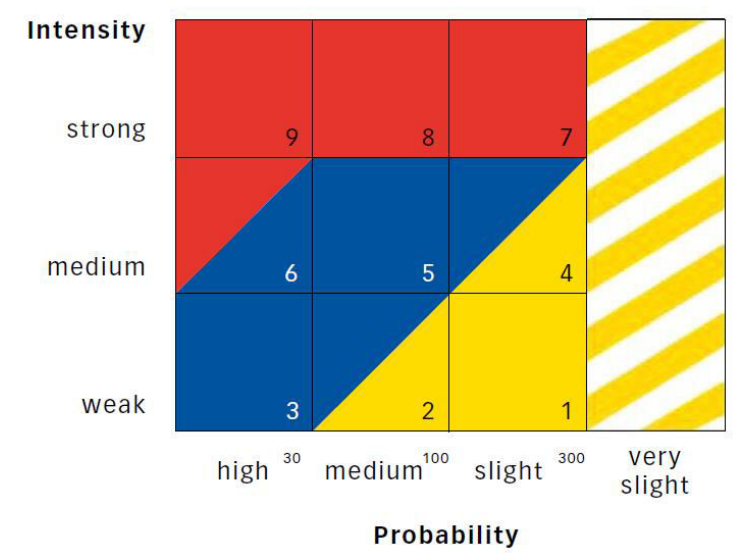

Figure 1. Assessment matrix for the identification of hazard levels in Switzerland (red = high; blue = medium; yellow = low, yellow-white stripped $=$ residual, white $=$ no or negligible) $[11]$

\subsection{Data on buildings}

We generate a country-wide comprehensive and homogenous data set of polygons of residential buildings and their period of construction by combining two data sets of (a) a topographic landscape model and (b) point data on residential buildings. From the 'Topographic Landscape Model TLM3D' provided the Federal Office of Topography ([12], version 1.1, 2012, TLM hereafter), we extract the feature group 'buildings', which contains footprints of all present buildings in Switzerland. These over two million footprint polygons "are not cartographically generalized and provide an accuracy of between 0.2 and $1.5 \mathrm{~m}$ " [12]. The information on residential use and period of construction as of the end of 2012 is taken from the federal register of residential buildings and dwellings, also known as the 'Gebäude- und Wohnungsregister (GWR)'. This register is provided by the Swiss Federal Statistical Office and contains nearly 1.7 million point-referenced records of buildings (entrances) with residential use [13]. The lengths of the 12 construction periods in the GWR differ considerably (from 2 to 27 years). In order to minimize these differences, we combine the seven shortest periods (of lengths between two and five years) to three (of lengths of ten and twelve years), obtaining the following eight periods: (1) before 1919; (2) 1919 - 1945; (3) 1946 1960; four ten-years periods (4-7) between 1961 and 2000; and (8) 2001 - 2012.

We intersect the point data of GWR with the building footprint polygons in order to assign the information on construction periods to the building footprints. Points that do not match a building polygon exactly are snapped to the nearest polygon within a distance of $\leq 5 \mathrm{~m}$. In this way, every building polygon contains the features 'numbers of associated GWR points' and 'construction periods of the associated GWR points'. We reduce the polygon data set to buildings with residential utilization, i.e. to polygons with at least one associated GWR point and thus with information on construction period. We further delete polygons with inhomogeneous construction periods, and finally reduce the data set to polygons with a construction period of determined length (i.e. 1919 onwards).

\subsection{Exposure analysis}

The building footprint polygons - pre-processed and selected as described above - are intersected with the compiled and harmonized flood hazard maps as defined by Fuchs et al. [6]. Every building is thereby classified to the highest hazard level it is intersecting with. For each of the eight time periods analysed, the total number (and annual average) of the constructed buildings and the share of exposed buildings (at hazard levels 'low', 'medium' and 'high') are calculated. We thus define 'share of exposed buildings' as the ratio of the number of 'newly constructed building that are exposed to floods (at respective hazard level)' to the number of 'total newly constructed buildings for which a flood hazard map exists.' We compute the ratio separately for each time period. Furthermore, we compare the exposure ratios of the three most recent time periods (1981-1999, 1991$2000,2001-2012)$ to the ones of the 1971 - 1980 time period. Finally, we present the results on timelines, indicating temporal evolution of flood exposure. For the analysed time period, we therefor assume that no building has been demolished nor replaced, and that the flood hazardous areas remained unchanged.

\section{Results}

The numbers resulting in the pre-processing of building data are presented in section 3.1, followed by the results of the flood exposure analyse in section 3.2. 


\subsection{Data set of building polygons including period of construction}

$1,464,978$ of the total $1,670,540$ data points of residential buildings and dwellings in GWR lay within a TLM building footprint polygon, and an additional 131,622 are within a distance of $\leq 5 \mathrm{~m}$. Consequently, $1,596,600$ (or $95.6 \%$ of the total $1,670,540$ ) data points are associated to a building footprint polygon.

Among the 2,053,539 TLM building footprint polygons $1,268,553(61.7 \%)$ are classified as buildings with residential utilization, i.e. at least one GWR data point is associated to them. The deletion of the 50,270 polygons with inhomogeneous construction periods reduces the data set further to 1,218,283 polygons. Finally, the 239,619 polygons from the construction period 'before 1919' are removed, resulting in a data set of 978,664 footprint polygons of buildings with residential utilization and homogenous construction periods between 1919 and 2012. These 978,644 building footprint polygons are used for the exposure analysis.

\subsection{Flood exposure}

In Switzerland, a flood hazard map exists for 691,529 (70.7\% of total 978,664 , see Tab. 1) residential buildings constructed between 1919 and 2012. That is, the areas where these buildings are located were part of the study areas of the cantonal hazard mapping procedures. Therefore, these buildings were assessed with respect to flood exposure. Of the assessed buildings, 110,745 $(16.01 \%)$ are exposed to floods. The exposure is mainly at hazard level 'low' (63,318 buildings or $9.16 \%)$. But some are also exposed considerably at level 'medium' (41,007 buildings or $5.93 \%$ respectively), whereas their exposure at hazard level 'high' is comparatively low with just 6,420 buildings $(0.93 \%)$ at that level.
In terms of annual newly constructed buildings, the period 1971-1980 shows the highest amounts, in 'all analysed buildings' (column 1 in Tab. 1) as well as in 'all buildings assessed in a flood hazard map' (column 2 in Tab. 1 and grey dashed line in Fig. 2). Yet, all exposure ratios (i.e. the number of newly constructed buildings within hazardous areas compared to the total number of newly constructed buildings within the perimeter of a flood hazard map) during this period of construction are lower than in any other period. Generally, it is remarkable that the amount of annual newly constructed buildings and the share of exposed buildings show a negative correlation (Fig. 2). When interpreting the data of consecutive time periods as time series, one detects a decrease in exposure ratios at hazard levels 'low' and 'medium' (and consequently summarized over all hazard levels) from 1919 to 1970 , whereas from 1981 to 2012 the same exposure ratios increase.

Comparing the exposure of the three time periods between 1981 and 2012 to the exposure rates of the time period 1971-1980, we notice remarkable differences between the three hazard levels considered (Fig. 3 and the last for columns in Tab. 1). While the exposure rates at hazard levels 'low' and 'medium' show comparable increases by factors 1.06 to 1.16 , the change at hazard level 'high' is strikingly higher up to factor 1.5 for the period 2001-2012, resulting from exposure ratios at hazard level 'high' of 1.11 (period 2001-2012) compared to 0.74 (period 1971-1980).

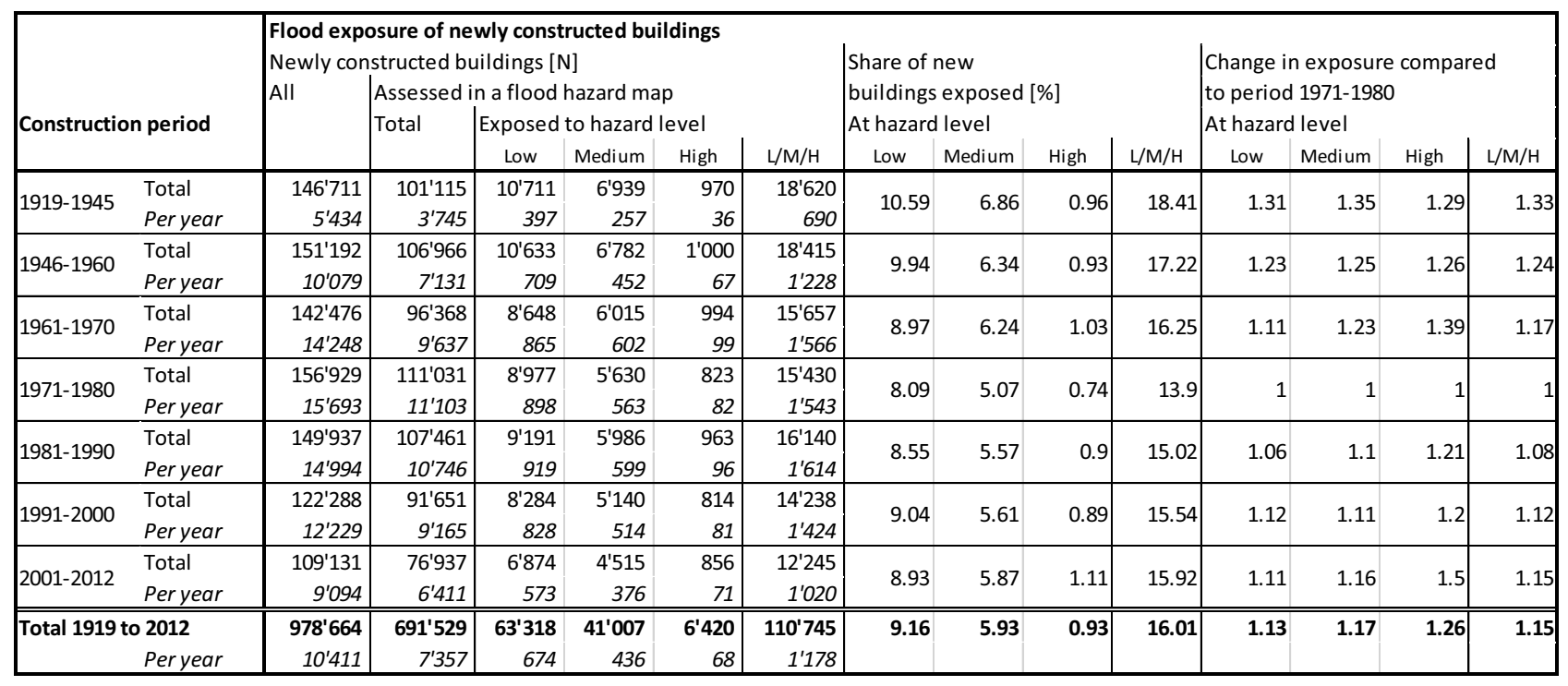

Table 1. Information on flood exposure of Swiss residential buildings constructed between 1919 and 2012. Hazard levels are explained in text, section 2.1. L/M/H = total exposure, sum of exposure to hazard level 'low' (L), 'medium' (M) and 'high' (H). The 'share of exposed buildings' (and the absolute number of annual newly built buildings assessed in terms of flood exposure, column 2) are additionally shown in Fig. 2, whereas the changes in exposure of time periods 1981-2012 compared to period 1971-1980 (last fozur columns) are illustrated in Fig. 3. 


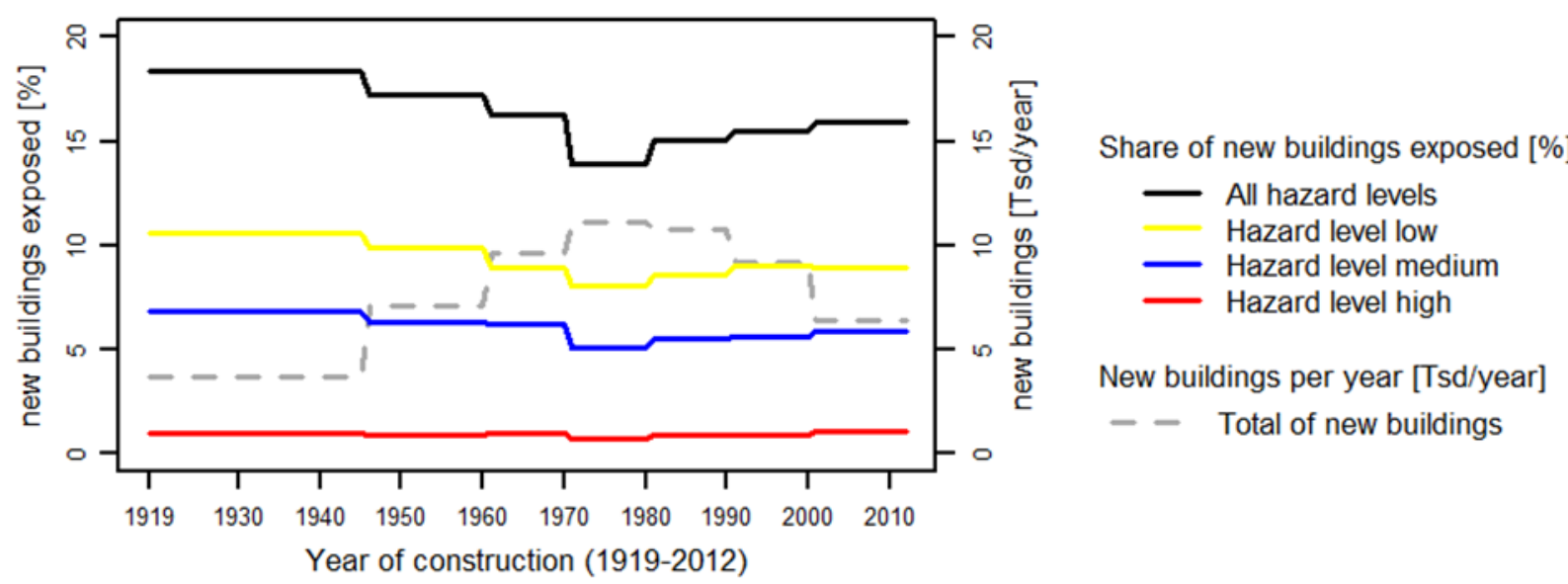

Figure 2. Newly constructed buildings per construction period. The 'share of new buildings exposed' is the ratio of the number of 'newly constructed building that are potentially exposed to floods (at respective hazard level)' to the number of 'total newly constructed buildings for which a flood hazard map exists'. The 'new buildings per year' are calculated by dividing the total number of buildings newly constructed within a particular time period by the length of the respective time period, figures are presented in thousands of buildings per year.

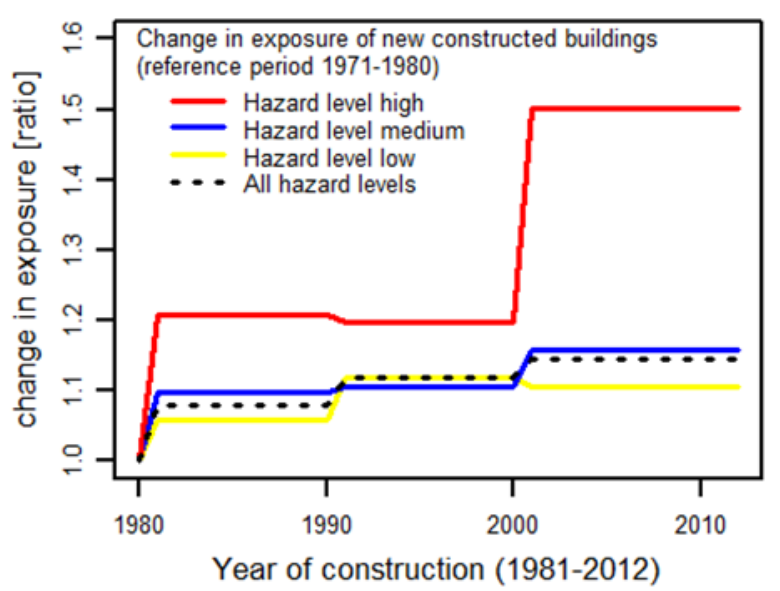

Figure 3. Relative change of the exposure ratios of the three most recent time periods (1981-1999, 1991-2000, 2001-2012) compared to the ones of $1971-1980$.

\section{Discussion and Conclusion}

When interpreting this study's results, one needs to keep in mind that we derive a development in the past from current data. Therefor, we assume that (a) flood threat areas remained constant and (b) no building was demolished nor replaced during the analysed periods of time.
Concerning the first assumption, it can be stated that extensive river trainings in Switzerland were completed by the end of the $19^{\text {th }}$ century, i.e. before the time period investigated in this study. Further on, recent structural protection measures are designed for events up to a 100-year return period. That means that 300-year events (and thus the encasing area of the analysed hazardous zones at levels 'low', 'medium' and 'high') are not influenced by these protection measures. However, the lines between areas of different hazard levels may be shifted. Therefore, temporal changes in the exposure to one specific hazard level must not be over-interpreted. In addition, the reliability of temporal comparisons of exposure ratio at different hazard levels decrease with the length of time period considered.

The second assumption (no demolition nor replacement of buildings) is less problematic in Switzerland than in other countries because Switzerland has (a) not been involved in any wars since the middle of the $19^{\text {th }}$ century and (b) standards in building construction and maintenance are comparatively high resulting in long times of use. As discussed in other publications [5, 6], however, studies on temporal dynamics that are based on current data records of construction year should focus on relative trends rather than on absolute values. Nevertheless, the presented absolute figures of (annual) newly constructed buildings and especially their relative changes over time are plausible. While the increase up to the 1970s may be explained by demographic parameters, the decrease after 1990 may be caused by the global financial crisis of the 1990s.

Looking at exposure rates, our findings of increasing rates since the $1980 \mathrm{~s}$ do not mirror the Switzerland-wide declared paradigm change "from protection against hazard to the management of risk" [14, front page] in the aftermath of the 1987 floods. It implies spatial planning as an imported part of risk management. In particular, the relatively high increase at hazard level 'high' is surprising as, for areas of this hazard level, the 
federal recommendations (and many cantonal and communal regulations) foresee a ban on construction of new buildings [11]. We see two possible explanations for this mismatch: time lag and obstacles to the enforcement in spatial planning processes. Thus, at national level, regulations on spatial planning (including the consideration of natural hazards) were introduced in the 1970s [15]. More details on natural hazards are regulated in the national laws on forest and on water [16, 17], which were totally revised and passed in the early 1990s. However, ten years later the federal extra-parliamentary commission for natural hazard PLANAT noted that "their implementation encounters very difficult conditions" [14:6].

Another remarkable result of the present study is the negative correlation between number of annual newly constructed buildings and the share of exposure. Sound statistical analyses as well as exploration of the underlying reasons of this phenomenon go beyond the scope of this paper, but are definitively worth examining in the future.

In conclusion, this study shows that nation-wide analysis of flood exposure at single building level is possible, and it derives new insights for the evaluation of risk management strategies. The temporal evolution of flood exposure ratios, with its lowest values in the 1970s, calls into question the effects of the risk management strategies introduced during the past decades and indicates a need for further investigation. Studies on exposure as one risk driver help to evaluate our risk management strategies and provide one basis for the improvement of risk management policies and practice. A comparison between different nation-wide exposure data and their development would add even more value to flood risk management.

\section{References}

1. Keiler M. (2013). World-wide trends in natural disasters. Encyclopedia of Natural Hazards, edited by: Bobrowsky, P., Springer, 1111-1114.

2. CRED [Centre for Research on the Epidemiology of Disasters] (2016). The OFDA/CRED international disaster database EM-DAT, Université Catholique de Louvain, Brussels, www.emdat.net, last access 26 February 2016.

3. IPCC [Intergovernmental Panel on Climate Change] (2012). Managing the Risks of Extreme Events and Disasters to Advance Climate Change Adaptation. A Special Report of Working Groups I and II of the Intergovernmental Panel on Climate Change, edited by: Field C. B., Barros V., Stocker T. F., Qin D., Dokken D. J., Ebi K. L., Mastrandrea M. D., Mach K. J., Plattner G.-K., Allen S. K., Tignor M., and Midgley P. M. Cambridge, UK, and New York, NY, USA, $582 \mathrm{pp}$.

4. Jongman B., Ward P.J. and Aerts J.C.J.H. (2012). Global exposure to river and coastal flooding: Long term trends and changes. Global Environmental Change, 22, 823-835.
5. Jongman B., Koks E.E., Husby T.G. and Ward P.J. (2014). Increasing flood exposure in the Netherlands: Implications for risk financing. Natural Hazards and Earth System Sciences, 14, 1245-1255.

6. Fuchs S., Keiler M., and Zischg A. (2015). A spatiotemporal mutli-hazard exposure assessment based on property data. Natural Hazards and Earth System Sciences, 15, 2127-2142.

7. BAFU [Bundesamt für Umwelt] (2015). Naturgefahrenkartierung der ganzen Schweiz steht. Press release of 26 May 2014. http://www.bafu.admin.ch/dokumentation/medienin formation/00962/index.html?lang=de\&msg$\mathrm{id}=53122$, last access 26 February 2016.

8. Hausmann P., Kunz Ch. and Rebuffoni G. (2012). Floods in Switzerland - an underestimated risk. Swiss Re Logistics Media Production. Zurich.

9. BWW [Bundesamt für Wasserwirtschaft], Bundesamt für Raumplanung BRP, Bundesamt für Umwelt, Wald und Landschaft BUWAL (1997). Berücksichtigung der Hochwassergefahren bei raumwirksamen Tätigkeiten.

10. BUWAL [Bundesamt für Umwelt, Wald und Landschaft] (1999): Risikoanalyse bei gravitativen Naturgefahren, Methode. Umwelt-Materialien Nr. 107/I.

11. ARE [Bundesamt für Raumentwicklung], Bundesamt für Wasser und Geologie BWG, Bundesamt für Umwelt, Wald und Landschaft BUWAL (2005): Empfehlungen Raumplanung und Naturgefahren / Federal Office for Spatial Development, Federal Office for Water and Geology, Swiss Agency for the Environment, Forests and Landscape: Recommendation Spatial Planning and Natural Hazards.

12. Swisstopo [Federal Office of Topography] (2012). swissTLM3D, large-scale topographical landscape model of Switzerland. Version 1.1 as per 2012. Description and acquisition on http://www.swisstopo.admin.ch/internet/swisstopo/e n/home/topics/geodata/TLM.html, last access 26 February 2016

13. Bundesamt für Statistik BfS (2008): Gebäude und Wohnungsregister, Merkmalskatalog / Federal Register of Buildings and Dwellings, Catalogue of attributes, Version 3.4.

14. National Platform for Natural Hazards PLANAT (2005). From protection against hazards to the management of risk. $19 \mathrm{p}$.

15. Schweizerische Eidgenossenschaft (1979). Bundesgesetz über die Raumplanung. SR 700.

16. Schweizerische Eidgenossenschaft (1991a). Bundesgesetz über den Wald. SR 921.0.

17. Schweizerische Eidgenossenschaft (1991b). Bundesgesetz über den Wasserbau. SR 721.100. 